\title{
High Resolution Millimeter Wave Images of HL Tau Reveal a Companion in the Disk
}

\author{
William J. Welch \& Zodiac Webster \\ University of California, Astronomy Department, Berkeley, CA 94720, \\ U.S.A.
}

Lee Mundy \& Nikolaus Volgenau

University of Maryland, Astronomy Program, College Park, MD 20742, U.S.A.

\author{
Leslie Looney \\ University of Illinois, Astronomy Department, 1002 W. Green St, \\ Urbana, IL 61801, U.S.A.
}

\begin{abstract}
An image of the disk of the TTauri star HL Tau at $1.4 \mathrm{~mm}$ wavelength at an angular resolution of 0.25 arcsec shows a secondary peak in the disk at a radius of $70 \mathrm{AU}$ and position angle of $-40^{\circ}$. This secondary peak is evidently a small unresolved nebulosity which may surround a star or planet or may not yet have formed such a body. The nebulosity could have formed as a result of a density wave instability in the disk.
\end{abstract}

\section{Introduction}

One of the most important areas of investigation relevant to the general question of origins concerns the study of the formation of stars and planets. While much is now known about the circumstances of star formation, many basic questions remain unanswered, such as: What determines the mass that a forming star will have?, and; What generally determines the Initial Mass Function (IMF) of the stars? In addition, we do not understand the frequency or the basic mechanisms for the formation of planets. The earliest stages of star and planet formation take place in the dense cores of molecular clouds, and the study of these processes must be made at long wavelengths at which the cloud cores are semi-transparent. Sub-arc-second angular resolution is also required.

The emission from the relatively cool outer parts of protostellar disks, where planets form, must be studied at millimeter or sub-millimeter wavelengths. For this purpose, the BIMA Millimeter Array has been recently equipped with new stations which provide baselines of up to $2 \mathrm{~km}$ and therefore angular resolution as high as 0.15 arcsec at $1.2 \mathrm{~mm}$ wavelength. A recent continuum BIMA image of the dust emission from the luminous embedded $\mathrm{T}$ Tauri star HL Tau at a wavelength of $1.4 \mathrm{~mm}$ provides a picture of the dusty disk around the star. The disk is largely symmetric with respect to the central star. In addition, there is a secondary peak in the brightness distribution at a radius of about $70 \mathrm{AU}$ in the disk. This secondary peak represents a nebulosity which could surround a second star or planet, or it could be such a body in the process of formation. 


\section{Observations}

The HL Tau observations employed the BIMA A- Array on December 26 of 1999 in the continuum at $1.4 \mathrm{~mm}$ wavelength (Welch et al. 1996). This array has a longest baseline of $1.3 \mathrm{~km}$ and has a diffraction limited resolution of about 0.25 arcsec at $1.4 \mathrm{~mm}$. The array uses nine antennas at this wavelength. The maximum angular structure that this array is sensitive to is about 2 arcsec which is adequate to image the entire extent of the HL Tau disk. Weather conditions were good with clear skies, $7 \mathrm{~mm}$ of precipitable water vapor overhead corresponding to an optical depth of 0.32 , and an RMS delay path difference of 135 micrometers on the $100 \mathrm{~m}$ baseline phase monitor. The system temperature averaged over all the receivers and all elevations was $450 \mathrm{~K}$ (SSB). The continuum IF bandwidth was $600 \mathrm{MHz}$ in each receiver sideband.

The observing strategy consisted of a series of three observations rapidly switched between HL Tau and the phase calibrator, either $0530+135$ or $3 \mathrm{C} 111$, followed by an observation of $0449+113$, also rapidly switched with the phase calibrator. Time on the source for each observation was $34.5 \mathrm{sec}$, with $23 \mathrm{sec}$ on the phase calibrator. Total time for the sequence was about 230 seconds, including time for the antenna slewing. This sequence was repeated over a total of about 7.8 hours. The rapid switching at half minute time scales removes some of the larger scale atmospheric phase fluctuations. The combination of the switching and the low atmospheric delay fluctuations observed on the phase monitor results in good image quality for the observations. $0449+113$ served as a test object for the experiment. Its image was constructed in the same way as that of HL Tau, which serves two purposes. The fact that its size corresponds to the expected synthesized beam means that features in the image of HL Tau can be trusted. Also, its apparent location in the field gives a direct measure of the accuracy of the angular registration of the HL Tau image since its absolute position is known with an accuracy of a few milliarcseconds (Perley 1982). No self-calibration was applied to either source. MWC349 with an adopted flux of $1.8 \mathrm{Jy}$ provided overall amplitude calibration, and the final total flux uncertainty is about $20 \%$.

\section{The HL Tau Image}

Figure 1 shows the $1.4 \mathrm{~mm}$ map of HL Tau as contours superposed on the $1.1 \mu m$ HST image. The image processing used Miriad (Sault, Teuben, \& Wright 1995). The only image deconvolution employed was CLEAN with a robustness parameter close to zero, leaving the same resolution as that of the dirty beam. The synthesized beam is 0.30 arcsec $\times 0.25$ arcsec with PA $=40^{\circ}$. The RMS noise in the map measured in the field is $5.0 \mathrm{mJy} / \mathrm{beam}$. The contours are separated by 2.3 sigma, beginning with the 2 sigma contour. The central peak is $100 \mathrm{mJy}$ and that of the secondary peak is $23 \mathrm{mJy}$. The secondary peak is at a position angle of $-40^{\circ}$ relative to the central source and at an angular separation of 0.5 arcsec, corresponding to a distance of $70 \mathrm{AU}$ for the assumed distance of 140 pc for L1551, the molecular cloud containing HL Tau. The secondary peak is unresolved. The $1.4 \mathrm{~mm}$ map is consistent with the earlier BIMA $2.7 \mathrm{~mm}$ maps (Mundy et al. 1996; Looney et al. 2000) but the overall extent seems a little smaller due to its better resolution. The disk appears inclined at an angle of about $45^{\circ}$ with its major axis having a position angle of about $-40^{\circ}$. 


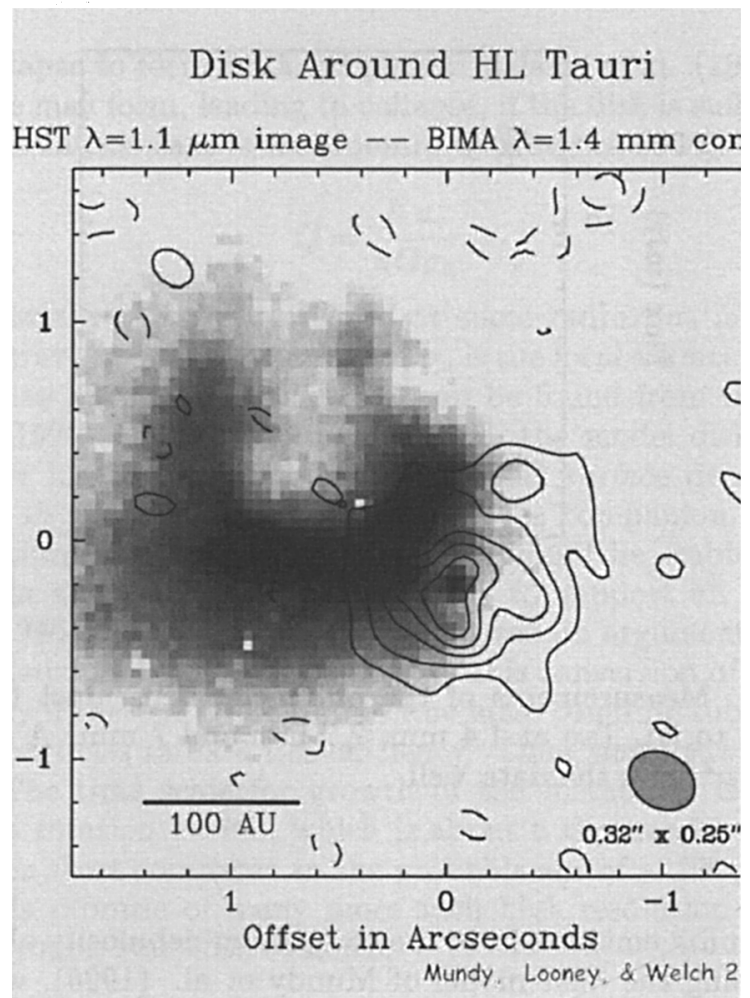

Figure 1. Image of HL Tau. The contours are the $1.4 \mathrm{~mm}$ continuum emission from the disk. The gray scale is the HST $1.1 \mu \mathrm{m}$ image.

The extent of the optical image and its displacement from the continuum millimeter contours of the disk shows that it is light scattered from nebulosity above the disk (Stapelfeldt et al. 1995; Close et al. 1997). The true $1.1 \mu \mathrm{m}$ stellar image appears faintly in the center of the millimeter contours. Based on the apparent position of the test source, $0449+113$, after phase calibration and mapping, we estimate the position of the millimeter HL Tau image to be registered to an absolute accuracy of 0.05 arcsec.

\section{Discussion}

The peak of the companion source is $4.6 \sigma$, which argues that it is a real feature. The $2.7 \mathrm{~mm}$ map of Looney et al. (2000) shows a hint, but not strong evidence of it because of poorer resolution. However, the $7 \mathrm{~mm}$ map of Wilner \& Lay (2000) definitely shows an extension in the same position with a flux of approximately $0.6 \pm 0.2 \mathrm{mJy}$. The $2.7 \mathrm{~mm}$ map shows a flux of $4 \pm 1 \mathrm{mJy}$ at the same position. Figure 2 shows a plot of the spectrum based on these three measures. The spectral shape is $\nu^{2.5}$, the same as for the overall source. The significant central disk opacity requires a dust emissivity index of $\beta=1$ along with the plank function to give this index for the flux of the overall source. However, the companion fluxes are all optically thin, so that $\beta=0.5$ for the companion, which suggests grain growth in its material. 


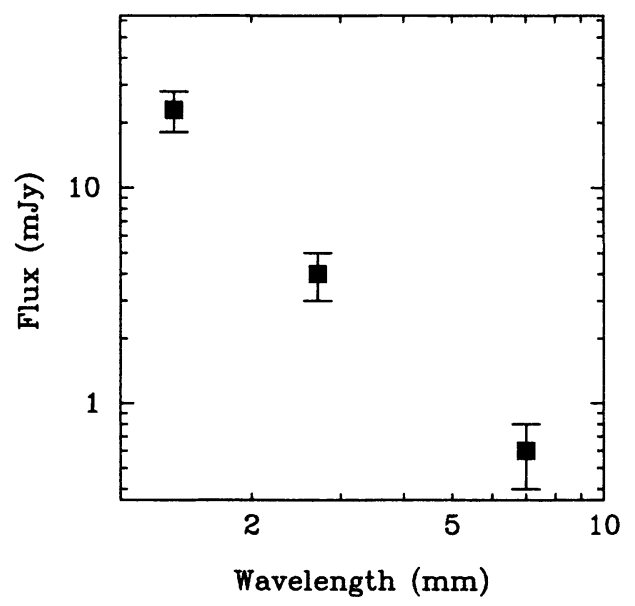

Figure 2. Measurements of the millimeter wave dust fluxes of the companion to HL Tau at $1.4 \mathrm{~mm}, 2.7 \mathrm{~mm}$ and $7 \mathrm{~mm}$. A straight line with slope $\nu^{2.5}$ fits the data well.

The continuum emission from the companion nebulosity allows an estimate of its mass. Using the dust model of Mundy et al. (1996), we obtain a mass of $.002 M_{\odot}$, about twice that of Jupiter, for the companion nebulosity. This mass is rather uncertain, and if there is grain growth, the mass may be higher (Miyake \& Nakagawa 1993). The reason is that larger grains radiate less well. If the companion were now in the process of collapsing to form a dense object, it appears that an object of planetary mass would result. On the other hand, collapse may have been underway for a while, and either a planet or, perhaps, a second star may be present within the small cloud.

Close et al. (1997) have made adaptive optics images of HL Tau in the $\mathrm{J}, \mathrm{H}$, and $\mathrm{K}$ ' bands at 0.2 arcsec resolution. Especially at $\mathrm{K}^{\prime}$ band they find faint images near the edges of the disk detected by Mundy et al. (1996) at 2.7 $\mathrm{mm}$ along the position angle of $125^{\circ}$. The intensity is about $1 / 300$ that of the central HL Tau brightness. This excess brightness is apparent in their trace of brightness along the major axis of their overall image, their Fig. 8. It seems likely that the nebulosity that we have detected corresponds to the excess brightness in their K' band image, and our radius is consistent with their intensity profile. They suggest that what they observe at $K^{\prime}$ is scattered light from the central star. Since we see an unresolved peak on one side of the star, it seems likely to be a single object. If it were self luminous rather than extended dust reflecting the light of the central star, its luminosity can be estimated from the data of Close et al. (1997) to be about 14.2 magnitude at K'. This would approximately correspond to an M8 dwarf star. Further high resolution observations at 3 and $10 \mu \mathrm{m}$ would help to distinguish among these possibilities.

One important question concerns the way in which the companion nebulosity may have formed. Adams et al. (1989) and Boss (2000) have discussed the possibility that a density wave instability in the disk around the central star 
may lead to collapse to form a star or planet. Adams et al. (1989) note that an $\mathrm{m}=1$ axial mode may form, leading to collapse, if the disk is sufficiently massive. A key parameter in this case is the Toomre Q (Toomre 1964).

$$
Q=\frac{K a_{\circ}}{\pi G \sigma_{\circ}}
$$

where $k$ is essentially the orbital period at some radius; $a_{\circ}$ is the local sound speed; $\mathrm{G}$ is the gravitational constant; and $\sigma_{\circ}$ is the local surface density. If $\mathrm{Q} \leq 1$, an instability may grow. The value of $Q$ can be found from the disk model of Mundy et al. (1996). At the radius of $70 \mathrm{AU}$, the model disk temperature is $43 \mathrm{~K}$. The other important number is the model surface density of the disk, which is about $15 \mathrm{~g} / \mathrm{cm}^{2}$. At the position of the companion, we find $\mathrm{Q}=3.5$. Although this number suggests that the disk should be stable, the disk mass is very uncertain and could be enough higher to support an instability. The Mundy et al. (1996) flux/mass conversion is based on arguments of Beckwith \& Sargent (1991), who assign an uncertainty to this conversion of at least a factor of 5. Particularly if there is grain growth, the mass could be substantially higher because larger particles radiate less efficiency. Thus, density wave instability is a possibility. The time scale for growth of the instability is expected to be comparable to a rotation period, which is about a thousand years in this case. This time scale is short compared to the probable age of $\sim 10^{5}$ years for HLTau. The future holds promise of many more such high resolution millimeter wave observations of young star/disk systems.

\section{References}

Adams, F. C., Ruden, S. R., \& Shu, F. H. 1989, ApJ, 347, 959

Beckwith, S. V. W., \& Sargent, A. I. 1991, ApJ, 381, 250

Boss, A. P. 2000, AAS/DPS, 32, 5209B

Close, L. M., Roddier, F., Northcott, M. J., Roddier, C., \& Graves, E. J. 1997, ApJ, 478, 766

Looney, L., Mundy, L., \& Welch, W. J. 2000, ApJ, 529, 477

Miyake, K., \& Nakagawa, Y. K. 1993, Icarus, 106, 20

Mundy, L. G., et al. 1996, ApJ, 464, L169

Perley, R. 1982, AJ, 87, 859

Sault, R. J., Teuben, P. J., \& Wright, M. C. H. 1995, in ASP Conf. Ser. Vol. 77, Astronomical Data Analysis Software and Systems IV, ed. R. Shaw, H. E. Payne, \& J. J. E. Hayes, 433

Stapelfeldt, K., et al. 1995, ApJ, 449, 888

Toomre, A. 1964, ApJ, 139, 1217

Wilner, D. J., \& Lay, O. P. 2000, in Protostars \& Planets, IV, ed. V. Mannings, A. P. Boss \& S. S. Russel (Tucson: University of Arizona Press), 509

Welch, W. J., et al. 1996, PASP, 108, 93 


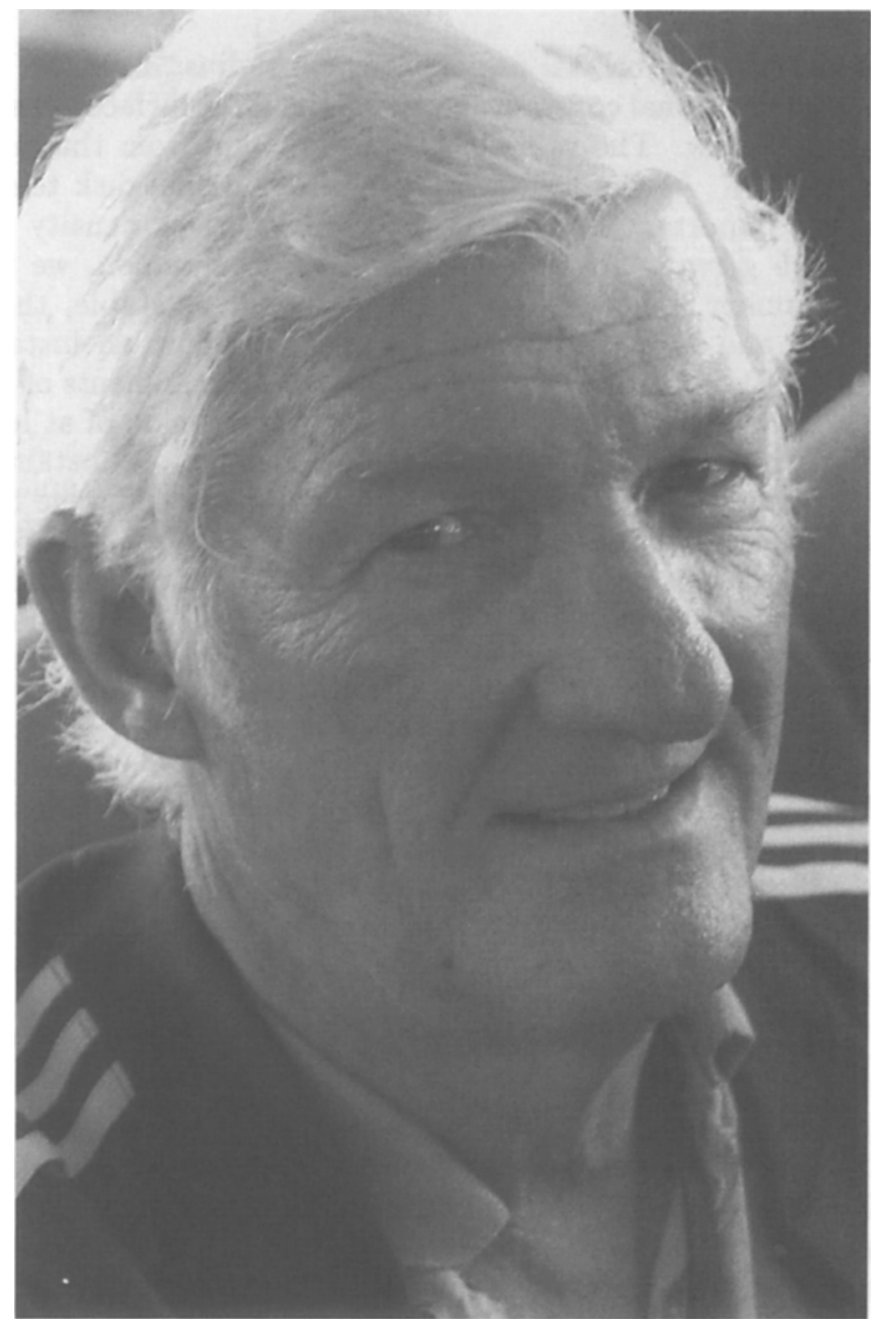

William (Jack) Welch (photo: Seth Shostak) 